\section{Efeito do treinamento resistido sobre a osteoporose após a menopausa: estudo de atualização}

\section{Effect of resistance training on postmenopausal osteoporosis: update}

\section{Resumo}

Objetivo: Investigar o efeito de intervenções com treinamento resistido sobre a força muscular e densidade mineral óssea nos sítios de maior ocorrência de fraturas relacionadas a osteoporose em mulheres no estágio de vida após a menopausa. Metodologia: Estudo de atualização por meio de revisão sistemática de ensaios controlados randomizados e meta-análise nas bases de dados do Colégio Americano de Medicina Esportiva e da Biblioteca Cochrane no período compreendido entre os anos de 1985 e fevereiro de 2005. Resultados: Foram encontrados vinte e seis estudos que atenderam os critérios de inclusão, realizados nos países Alemanha, Austrália, Áustria, Canadá, China, Estados Unidos, França, e Japão, com um total de 2.300 mulheres com idades entre 40 e 92 anos. Intervenções com treinamento resistido apresentaram resultados estatisticamente significantes sobre a força muscular e a densidade mineral óssea nos sítios vértebras lombares, fêmur (triângulo de Ward/trocanter) e quadril total. Conclusão: $\mathrm{O}$ treinamento resistido mostrou ser capaz de prover estímulo para aumentar a força muscular e a formação óssea, influenciando os fatores de risco relacionados com osteoporose e quedas seguidas de fratura em mulheres no estágio de vida após a menopausa.

Palavras-chave: Treinamento resistido. Osteoporose após a menopausa. Densidade mineral óssea. Ensaios controlados randomizados. Meta-análise.

Agradecimentos: Aos mestres e funcionários da Faculdade e Biblioteca de Saúde Pública da Universidade de São Paulo e do Centro Cochrane do Brasil, e aos revisores da Revista Brasileira de Epidemiologia, pelos apontamentos que muito contribuíram para a elaboração do texto final deste manuscrito.

Correspondência: Marcia Salavar Jovine. Rua Simpatia, 160 apto. 640 - Vila Madalena - São Paulo, SP CEP 05436-020.E-mail: m@ajato.com.br 
Abstract

Objective: to investigate the effect of interventions with resistance training on muscular strength and bone mineral density on the sites of higher incidence of osteoporotic fractures in postmenopausal women. Methodology: update study through a systematic review of randomized controlled trials and meta-analysis in the databases of the American College of Sports Medicine and the Cochrane Library was conducted from 1985 up to, and including, February 2005. Results: twenty-six studies met inclusion criteria from Germany, Australia, Austria, Canada, China, United States, France and Japan, with a total of 2,300 women with ages ranging from 40 to 92 years. Interventions with resistance training showed statistically significant results on muscular strength and bone mineral density of the lumbar spine, femur (Ward/ trochanter) and total hip. Conclusion: resistance training markedly influences risk for osteoporotic factors regarding falls (muscular strength) and bone mineral density in postmenopausal women.

Keywords: Resistance training. Osteoporosis. Postmenopausal women. Bone density. Randomized controlled trial. Meta-analysis.

\section{Introdução}

Dentre os declínios funcionais do processo de envelhecimento, a redução de densidade mineral óssea (DMO) assume particular importância pelo potencial desenvolvimento da osteoporose e por elevar o risco de fraturas em ambos os sexos ${ }^{1}$.

A osteoporose é considerada um grave problema de saúde pública em todo o mundo. Nos Estados Unidos, mais de 25 milhões de pessoas estão predispostas a 1,3 milhão de fraturas ao ano, com um custo provável da ordem de 10 bilhões de dólares $^{2}$, equiparando-se ao montante relativo a outras importantes doenças crônicas, como as cardiovasculares e asma, enquanto a diminuição de aproximadamente $15 \%$ na esperança de vida assemelha-se aos índices observados nos pacientes com doenças coronarianas ${ }^{3}$. No continente europeu, acomete $11 \%$ da população e $31 \%$ das mulheres acima dos 50 anos ${ }^{4}$.

A queda na produção dos estrogênios, característica do estágio de vida após a menopausa, é um fator que acelera a redução de $\mathrm{DMO}^{5,6}$. Isso faz das mulheres uma população especialmente suscetí$\mathrm{vel}^{7,8}$, e da osteoporose primária do tipo I ou após a menopausa, um grande interesse para a saúde pública ${ }^{1}$.

No Brasil, $10 \%$ da população feminina vive um terço ou mais do total de anos de vida após a menopausa e a meta de assistência a essa parcela inclui medidas com enfoque na mudança comportamental e investimento de longo prazo na saúde ${ }^{9,10}$.

Uma das medidas preventivas que destacamos é a atividade física, especialmente os exercícios que desencadeiam contrações musculares contra alguma forma de resistência externa, geralmente pesos, denominados em português, de: treinamento com pesos, ou de força, ou contra-resistência. Em inglês, as denominações clássicas são weight training, strenght training, ou mais recentemente, resistance training ou resistive training, cujo significado é treinamento de força realizado contra resistências ${ }^{11}$. 
$\mathrm{O}$ treinamento resistido (TR) quando praticado com regularidade, pode aumentar a força muscular com positivas repercussões na proteção contra as quedas, além do eficiente estímulo para o aumento da massa óssea ${ }^{12-15}$, influenciando fatores de risco relacionados com osteoporose.

Revisão realizada em $1999^{16}$ encontrou aproximadamente vinte estudos longitudinais e de corte transversal mostrando relação direta e positiva entre o efeito do TR sobre a DMO. No entanto, um número semelhante de estudos, reportava pequeno ou nenhum efeito sobre massa óssea. A divergência entre os resultados foi parcialmente atribuída ao pequeno número de participantes, intensidade e duração dos protocolos de exercícios, baixa adesão e uso de técnicas diferentes para mensuração de DMO.

Com o objetivo de investigar o efeito do TR na prevenção dos fatores de risco para osteoporose primária do tipo I, relacionados a quedas (força muscular) e DMO nos sítios de maior ocorrência de fraturas, realizamos um estudo de revisão dos trabalhos mais recentes, melhor elaborados, com controle mais rígido da metodologia a partir da consulta em bases de dados que apresentavam alta probabilidade de reunir os trabalhos de nosso interesse.

\section{Metodologia}

Para realizar este estudo de atualização, por meio de uma revisão sistemática, definimos a busca por ensaios controlados randomizados ${ }^{17}$ e meta-análises ${ }^{18}$, no período compreendido entre janeiro de 1985 e fevereiro de 2005.

A primeira consulta foi realizada no banco de dados do Colégio Americano de Medicina Esportiva (ACSM - American College of Sports Medicine $)^{19}$ - BASE A (Anexo I), por meio de seu periódico Medicine \& Science in Sports \& Exercise.

A estratégia para a procura baseou-se nos descritores usados pela Biblioteca Nacional de Medicina dos Estados Unidos: bone density, bone mineral density, weight training, resistance training, resistance exercise, osteoporosis, postmenopausal women $^{20}$. Os estudos foram analisados um a um.

A segunda consulta foi realizada na Biblioteca Cochrane e sua Base de Dados de Revisões Sistemáticas ${ }^{21}$ - BASE B (Anexo II).

A estratégia de busca foi pesquisar o Registro Cochrane de Ensaios do Grupo Músculo-esquelético, o Registro Cochrane de Ensaios Clínicos Controlados/Randomizados, a MEDLINE, (MEDlars on LINE, produzida pela Biblioteca Nacional de Medicina dos Estados Unidos), EMBASE (base de dados da Excerpta Médica, sediada na Europa), HealthStar, SportsDiscus, e Conteúdos Atualizados (das edições mais recentes das mais importantes publicações acadêmicas). Dentre as restrições enfrentadas, diante do fato de não encontrarmos referência específica a TR (como aconteceu no Índice Alfabético de Títulos de Revisões Cochrane $)^{22}$, houve a necessidade de selecionar os trabalhos que referissem intervenções com exercício físico de maneira generalizada (TR combinado com outros tipos de exercício físico), usando os descritores: bone density, bone loss, clinical trial, control ou controls ou controlled, doubleblind method, exercise, exercise therapy, osteoporosis, physical activity, physical fitness, placebos, random allocation, randomised controlled trial, single-blind method, sports.

Os estudos desta base de dados estão integrados em uma meta-análise (programa de computador RevMan 4.1) ${ }^{23}$.

\section{Critérios para exclusão de estudos nas duas bases de dados}

Foram excluídos os ensaios clínicos não randomizados, fora do período considerado, aqueles com intervenções que não incluíram TR, e os estudos que apresentaram desfechos outros que não a força muscular e a DMO.

Não houve restrição de idioma na pesquisa original. 


\section{Critérios para inclusão de estudos}

Para a seleção dos artigos que participaram dessa revisão, foram definidos critérios em relação às amostras, ao tipo de intervenção e à mensuração dos desfechos.

Assim, foram considerados na BASE A:

- amostras compostas por mulheres, entre os primeiro e oitavo anos após a menopausa, sedentárias há pelo menos seis meses antes da participação nos estudos, com idades entre 40 e 68 anos;

- intervenções que estudaram o efeito de programas de exercícios assumidos como adequados para incrementar a força muscular e a DMO: TR de alta e baixa frequência, TR de alta carga e alta repetição, TR de alta carga e baixa repetição, TRP (treinamento resistido progressivo), TR combinado com exercícios calistênicos (de sustentação do peso corporal), comparados àqueles assumidos como adequados para melhorar condicionamento cárdio-respiratório e endurance (exercícios aeróbios) e/ou grupos controle de não exercício;

- os desfechos de interesse observados em cada um dos estudos selecionados tiveram sua significância estatística avaliada de acordo com valor de $\mathrm{P}$ [0 a $1]^{17} \mathrm{e}$, respeitando protocolos padronizados no início e final dos estudos, incluíram:

- mensuração dos fatores relacionados com risco de quedas e fraturas por meio de testes padronizados: de força dinâmica (uma repetição com máxima carga - 1 RM), de máxima força isométrica (máquina Schnell M-3), de potência de membros superiores e inferiores (mensuradas respectivamente por arremesso de medicine ball à distância e picos em "watts" no cicloergômetro), de resistência absoluta (teste de 50 a $70 \%$ de $1 \mathrm{RM}$ repetido até a exaustão) e de flexibilidade (teste padrão "sentar e alcançar");

- mensuração de DMO, por meio de DXA (dual $\mathrm{x}$-ray absorptiometry) em $\mathrm{g} / \mathrm{cm}^{2}$ nos sítios: vértebras lombares, fêmur (colo/triângulo de Ward/trocanter), quadril total, porcentagem de mudança de DMO em todo o corpo e relação entre porcentagem de mudança de osteocalcina e DMO;

\section{e, na BASE B:}

- amostras compostas por mulheres no estágio de vida após a menopausa, saudáveis, com idades entre 45 e 92 anos;

- intervenções que estudaram o efeito de programas de exercícios assumidos como adequados para incrementar a força muscular (as sessões foram organizadas com exercícios de fortalecimento isotônico-concêntrico de grandes grupos musculares) e a DMO - TR de baixa e alta carga e TR combinado com exercícios aeróbios e calistênicos, comparados a grupos-controle de não exercício;

- os desfechos de interesse observados nos estudos integrados na meta-análi$\mathrm{se}^{23}$, tiveram sua significância estatística avaliada de acordo com intervalo de confiança [IC] com grau de certeza determinado em $95 \%{ }^{17}$ e, respeitando protocolos padronizados no início e final dos estudos, incluíram:

- mensuração de DMO, por meio de "Single-Photon Absorptiometry", "Dual-Photon Absorptiometry" ou “DXA - Dual X-Ray Absorptiometry". Os sítios investigados foram: vértebras lombares, fêmur (colo/trocanter), quadril, quadril total, punho (antebraço) e porcentagem de mudança de DMO de todo o corpo.

\section{Considerações éticas}

A pesquisa foi realizada a partir de dados secundários, sendo de domínio público a identificação dos autores envolvidos. À parte isso, o Comitê de Ética em Pesquisa - COEP, concedeu parecer favorável para o desenvolvimento da mesma. 
Não houve conflito de interesses ou qualquer financiamento para a realização do estudo.

\section{Resultados}

- Nas duas bases de dados consultadas BASE A e BASE B (Anexos I e II), vinte e seis estudos atenderam os critérios préestabelecidos, realizados nos países Alemanha, Austrália, Áustria, Canadá, China, Estados Unidos, França e Japão; com um total de 2.300 participantes e idades entre os 40 e 92 anos.

- O efeito sítio-específico de cada intervenção sobre a força muscular e a DMO é apresentado de acordo com as diferentes bases de dados.

Na BASE A, de acordo com valor de P, as seguintes variáveis preditoras foram estatisticamente relacionadas como proteção para os riscos de quedas e fraturas: no estudo 02 (força dinâmica $[\mathrm{P}<0.05]$ ), no estudo 04 (força dinâmica e resistência absoluta $[\mathrm{P}=0.001]$, potência $[\mathrm{P}=0.002]$, e flexibilidade $[\mathrm{P}=0.017]$ ), no estudo 05 (força dinâmica e máxima força isométrica $[\mathrm{P}$ $<0.001$ ) ) e no estudo 07 (força dinâmica [P $<0.001]$ ). Os efeitos positivos mostraram ser parcialmente dependentes do volume do treinamento, da freqüência às sessões e total de peso levantado durante o tempo de seguimento das intervenções.

Em relação à DMO, os resultados que mostram associação positiva entre TR e DMO em mulheres no estágio de vida após a menopausa, segundo sítios, foram vértebras lombares (estudos 01 e $05[\mathrm{P}<0.001]$ ) e fêmur/triângulo de Ward (estudo $01[\mathrm{P}<$ 0.05]). O estudo 03 apontou uma relação positiva entre a mudança na porcentagem de osteocalcina e a mudança na porcentagem de DMO nos sítios fêmur/trocanter ([P $=0.04])$ e quadril total $([\mathrm{P}=0.048])$. No estudo 06 , os resultados sugerem a evidência de uma relação linear entre mudança de DMO, de acordo com a quantidade de peso levantado em cada exercício específico e a soma total de peso levantado em todos os exercícios. O efeito foi notado no sítio fêmur/trocanter e na porcentagem de mudança de DMO de todo o corpo ([P $<0.01])$. O exercício "agachamento" foi considerado como o que mais contribuiu para aumentar DMO de todo o corpo.

Comparando os grupos exercício e controle, houve perdas estatisticamente significantes de DMO, nestes últimos, nos sítios fêmur/colo (estudo $01[\mathrm{P}<0.05]$ ) e vértebras lombares (estudos $02[\mathrm{P}<0.05] \mathrm{e}$ $05[\mathrm{P}<0.001])$.

Na BASE B, de acordo com intervalo de confiança, os resultados mostram que houve efeito estatisticamente significante sobre a DMO no sítio vértebras lombares nos estudos $02,10,16$ e 18 (IC [+0.44 a $+4.57]$ ), e nos estudos 12 e 13 (IC [+0.58 a $+3.01]$ ).

Os efeitos observados nos sítios fêmur/ colo e quadril (estudos 12, 13 e 14 com IC [-1.18 a +1.03]), quadril total (estudos $10 \mathrm{e}$ 16 com IC [-0.85 a +1.67]) e punho (estudo 11 com IC [-3.2 a +2.65] e estudos 12 e 14 com IC [+0.71 a + 1.74]) foram positivos isoladamente, mas considerados estatisticamente não significantes quando integrados à meta-análise.

Destacando apenas os desfechos com significância estatística, a síntese desses dados está distribuída na Tabela 1 .

\section{Discussão}

Na BASE A, consideramos importante o efeito positivo do TR nos parâmetros relacionados com risco de quedas e fraturas, encontrados nos estudos 02 (força dinâmica), 04 (força dinâmica, resistência absoluta, potência e flexibilidade), 05 (força dinâmica e máxima força isométrica) e 07 (força dinâmica), porque mulheres no estágio de vida após a menopausa apresentam alto risco para sarcopenia (perda de função muscular típica do envelhecimento sedentário) ${ }^{24}$.

Os estudos 02, 03 e 04 (Anexo I), que aplicaram programas de intervenção de curta duração (2 a 6 meses) com TR de 60 a $90 \%$ de 1RM, resultaram efeito pouco sig- 
Tabela 1 - Efeito do tipo de exercício sobre a força muscular* e a DMO em sítios-específicos, segundo bases de dados. Table 1 - Effect of the type of exercise on strength* and BMD (bone mineral density) on specific sites, according to databases.

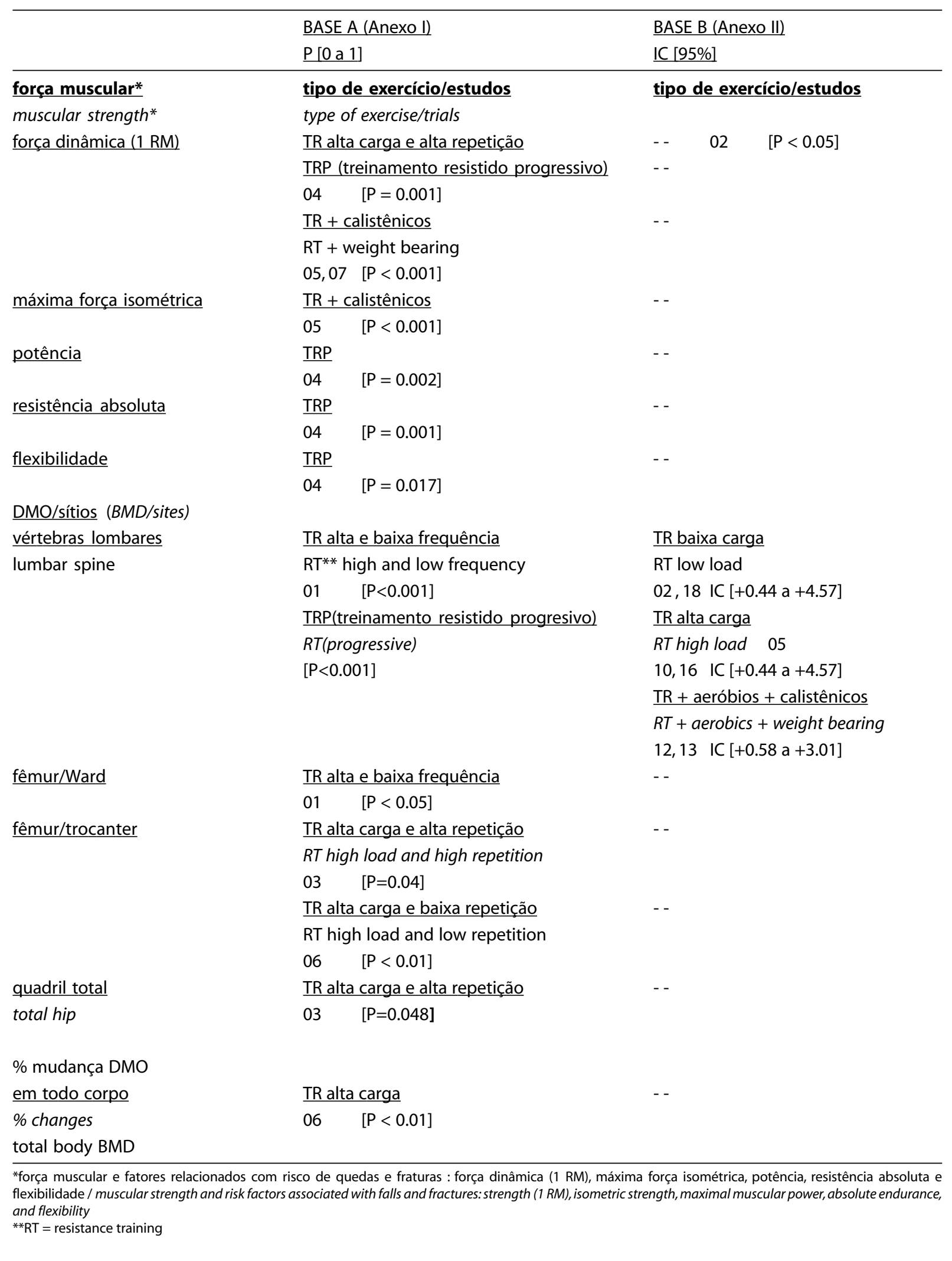


nificativo em DMO. Isso talvez seja explicado pelo fato de os valores iniciais (medidas de base) das participantes já ser alto e por amostras compostas também por mulheres que ainda não estavam no estágio de vida após a menopausa (idades variavam de 41 a 68 anos), uma vez que populações com baixos valores de DMO inicial em curtos períodos de tempo costumam apresentar melhores resultados do que aquelas com parâmetros iniciais mais altos $^{25,26}$. De acordo com princípios fisiológicos, os maiores benefícios provavelmente são alcançados para indivíduos com valores iniciais de DMO mais baixos. Estudos de curta duração apresentam limitação por causa dos ciclos de remodelação óssea durarem de 4 a 6 meses, o que pode alterar a interpretação dos desfechos.

O estudo 03 comparou protocolos de TR de alta intensidade e baixa repetição com os de baixa intensidade e alta repetição, desenhados para produzir semelhantes volumes de trabalho (séries versus repetições versus carga). Ambos resultaram em aumento de força muscular. Os protocolos de baixa intensidade e alta repetição apresentaram o benefício adicional de serem mais facilmente tolerados e com similar benefício para saúde e condicionamento físico. Embora não corroborada pelos achados que apontam para o estímulo mecânico de máxima carga ser mais importante que o número de ciclos de carga para extrair adaptações ósseas ${ }^{27,28}$, esta é uma informação muito valiosa para a prescrição de exercícios para mulheres sedentárias com osteopenia ou osteoporose, para as quais os exercícios de alta intensidade são contra-indicados.

No estudo 08, os achados apontam para o fato de o exercício estancar a perda de massa óssea em vértebras lombares. Ainda nesse estudo pequenos ganhos em força e resistência podem estar associados ao fato de não ter havido aumento do número e/ou duração das sessões semanais, além do aumento das cargas durante o período de intervenção (38 meses), embora a preocupação fosse evitar desistências e aumentar aderência para população sedentária. É preciso também considerar o princípio fisiológico da diminuição dos resultados, a respeito do fato de os indivíduos aparentemente terem um teto biológico/genético que determina a extensão da melhoria: os progressos do treinamento podem atingir esse teto e os incrementos, por outro lado, diminuem ou eventualmente estacionam.

Declínios na DMO foram observados no sítio fêmur/colo (estudo $01[\mathrm{P}<0.05]$ ) e no sítio vértebras lombares (estudos 02 $[\mathrm{P}<0.05]$ e $05[\mathrm{P}<0.001])$ nos grupos-controle que não receberam intervenções com TR. Quando se sabe que a perda óssea de $5 \%$ ao ano ${ }^{29}$ é comum em mulheres no estágio de vida após a menopausa, esses resultados encorajam futuros estudos implementando TR para redução da velocidade de remodelação óssea.

Na BASE B, os resultados da meta-análise mostraram que há alguma evidência do efeito das intervenções na redução da velocidade da perda óssea, quando realizadas por um ano ou mais.

Os programas só foram executados durante o período total de seguimento; então, não se pode concluir que eles são eficientes após a descontinuação da terapia, o que seria confirmado pelo princípio fisiológico da reversibilidade, segundo o qual nenhum efeito positivo de treinamento sobre DMO é mantido por longos períodos se o exercício não for continuado. Porém, sobre esse ponto particular, encontramos um estudo prospectivo que determinou o efeito protetor de longa duração da força dos músculos das costas sobre as vértebras lombares, oito anos após as pacientes terem completado a participação. Quando comparadas com grupo controle, as diferenças estatisticamente significantes perduraram por 10 anos nos parâmetros força muscular [ $\mathrm{P}=0.001]$, incidência de fratura por compressão vertebral $[\mathrm{P}=0.0290]$, e DMO - que não apresentou diferença significante entre os grupos nas medidas de base e após dois anos, mas foi significante no décimo ano $[\mathrm{P}=0.0004]^{30}$. 
Atualmente, o curto tempo de seguimento de grande número dos estudos limita nossa habilidade para melhor discutir os efeitos de longa duração que o exercício pode ter ou não sobre os ossos.

Os estudos referiram de maneira muito irregular os fatores de influência sobre a efetividade do exercício terapêutico, como freqüência e aderência ao programa, tipo de exercício, duração de cada sessão e intensidade dos exercícios, número de sessões semanais (1 a 5) e duração dos programas de exercícios em meses ( 2 a 60 ). Essa irregularidade limita conclusões que podem ser extraídas desta meta-análise, mesmo diante de resultados positivos. A heterogeneidade entre os estudos e a população incluída pode ser responsável por discrepâncias; também as conclusões poderão mudar se novos estudos forem incluídos no futuro.

Revisão conduzida na base de dados Medline, nos anos compreendidos entre 1979 e $1999^{31}$, concluiu, por evidências epidemiológicas, que nas populações em envelhecimento o efeito do exercício sobre DMO sugere que ser ativo fisicamente pode frear a incidência de fraturas de quadril, e que exercício praticado durante a vida mostra ser encorajador para maximizar pico de massa óssea, reduzir sua perda e o risco de quedas.

Nessa área também encontramos pequeno número de publicações de metaanálises. Assim como os achados na BASE B, Berard, Bravo e Gauthier, $1997^{32}$ conclu- íram que TR e exercícios calistênicos têm efeito sobre DMO principalmente no sítio vértebras lombares, e algum efeito nos sítios fêmur e antebraço, e Wolff, Kemper, Konstence e Twisk, $1999^{33}$ concluíram que exercícios previnem perda óssea femoral e de vértebras lombares em mulheres nos estágios de vida pré e pós-menopausa.

Essas observações são características de um assunto recente, que pede por variadas diretrizes para pesquisas futuras. Ao indicar tais diretrizes, o NIH (National Institute of Health) ${ }^{34}$ salienta a importância do alto pico de massa óssea como fator determinante para diminuir risco de fratura de longo termo, e que estratégias para maximizá-lo em jovens são essenciais. Identificar e intervir nos distúrbios que possam impedir essa aquisição, em diferentes etnias, além de determinar quantidade e duração dessas intervenções, é também uma das prioridades preventivas.

\section{Conclusão}

Os dados recolhidos nas BASES A e B, indicam a necessidade de padronização para o desenvolvimento de estudos de intervenção e informações de desfechos, de forma a garantir, em futuras pesquisas, a comparabilidade dos dados e, assim, poder determinar os mais eficientes métodos de educação para o público e profissionais de saúde a respeito de prevenção, diagnóstico e tratamento de osteoporose primária do tipo I.

\section{Referências}

1. Woolf AD. Bulletin of the World Health Organization. Disponível em URL: http:/ /www.who.int/docstore/ bulletin/editorials/issue5/strongbones. Acessado em 20 de novembro de 2004

2. Chan KM, Anderson M, Lau EMC. Exercise interventions: defusing the world's osteoporosis time bomb. Boletim da Organização Mundial da Saúde 2003; 81:827-30. Disponível em URL: http://www.who.int/bulletin/ volumes/81/11/en/mingchanwa1103. pdf . Acessado em 20 de novembro de 2004.
3. Consensus Development Conference. Diagnosis, Prophylaxis, and Treatment of Osteoporosis. Am J Med 1993; 94: 646.

4. Efort-European Federation of National Associations of Orthopaedis and Traumatology. S3041 Osteoporotic Fractures - Growing Problem, S3042 Spinal Fractures in Osteoporosis, S3043 Distal Radius Fractures. JBone and Joint Surgery 2004; 86-B (SIII): 218-21. 
5. Gracia CR, Sammel MD, Freeman EW, Lin H, Langan E, Kapoor S, Nelson DB. Defining menopause status: creation of a new definition to identify the early changes of the menopausal transition. Menopause 2005; 12(2): 128-35.

6. Aldrighi JM, Aldrighi CMS, Aldrighi APS. Alterações sistêmicas no climatério. Rev Bras Med 2002; 59: 15-21.

7. Girasole G, Jilka RL, Passeri G. 17b-estradiol inhibits interleukin- 6 production by bone marrow-derived stromal cells and osteoblastic in-vitro: potential mechanism for the antiosteoporotic effect of estrogens. $J$ Clin Invest 1992; 89:883.

8. Pottratz ST, Bellido T, Mocharla H, Crabb D, Manologas SC. 17b-estradiol inhibits expression of human interleukin-6 promoter-reporter constructs by a receptordependent mechanism. J Clin Invest 1994; 93: 994.

9. MS - Ministério da Saúde. Secretaria de Assistência à Saúde. Departamento de Assistência e Promoção à Saúde. Coordenação Materno-Infantil. Assistência ao Climatério. Brasília (DF); 1994. Disponível em URL: http:/ /www.vicnet.com.br/ starfire/sobrac/20.htm e Fundação IBGE, Censos Demográficos de 1900 a 1991 e Contagem Populacional de 1996; Frias LA de M, Carvalho JAM (1994) Censos demográficos de 1980 e 1991; SIM - DATASUS/ FNS; 1995. Acessado em 14 de outubro de 2005.

10. Waldman EA, colaboração Rosa TEC. Doenças crônicas não transmissíveis - Prevenção. Secretaria de Estado da Saúde - SP (SPS - Secretaria de Políticas de Saúde Saúde e Cidadania-Para gestores municipais em Saúde Pública). Vigilância em Saúde Pública, 1998. Disponível em URL: http://dltr2001.saude.gov.br/sps/dicas.htm. Acessado em 12 de fevereiro de 2005 .

11. Santarem JM. Fisiologia do exercício e treinamento resistido na saúde, na doença e no envelhecimento. Disponível em URL: http://saudetotal.com/cecafi/ texto.htm. Acessado em 20 de dezembro de 2003.

12. Greendale GA, Huang MH, Wang Y, Finkelstein JS, Danielson ME, Sternfeld B. Sport and home physical activity are independently associated with bone density. Med Sci Sports Exerc 2003; 35(3): 506-12.

13. Karam FC, Meyer F, Souza ACA. Esporte como prevenção de osteoporose: um estudo da massa óssea de mulheres pós menopáusicas que foram atletas de voleibol. Rev Bras Med Esporte 1999; 5(3): 86-92, tab. [LILACS].

14. Braith RW, Mills RM, Welsh MA, Keeller JW, Pollock ML. Resistance exercise training restores bone mineral density in heart transplant recipients. JAm Coll Cardiol 1996; 28: 1471- 7 .

15. Karlsson MK, Johnell O, Obrant KJ. Bone mineral density in weight lifters. Calcif Tissue Int 1993; 52: 212-5.

16. Layne JE, Nelson ME. The effects of progressive resistance training on bone density: a review. Med Sci Sports Exerc 1999; 31(1): 25-30.
17. The Cochrane Reviewers' Handbook Glossary. Version 4.1.2. The Cochrane Collaboration, updated 27 february 2002. Disponível em URL: http://www.cochrane.dk/ cochrane/handbook/htm. Acessado em 28 de agosto de 2005.

18. Glossário de Medicina Preventiva e Saúde Pública. Disponível em URL: http://www.fm.ul.pt/public/ Med_Preventiva/www/dicionario_dt.htm\#ErrotipoI. Acessado em 22 de janeiro de 2006.

19. ACSM. Disponível em URL: http://www.acsm.org/pdf/ endapp.pdf. Acessado em 20 de janeiro de 2006.

20. DeCS - Descritores em Ciências da Saúde. Disponível em URL: http://decs.bvs.br/. Acessado em 4 de fevereiro de 2005.

21. Biblioteca Cochrane. Disponível em URL: http:// cochrane.bireme.br/index.php. Acessado em 20 de janeiro de 2006.

22. Alphabetical list of titles of Cochrane Reviews. Disponível em URL: http://www.centrocochranedobrasil.org. Acessado em 28 de agosto de 2005.

23. Bonaiuti D, Shea B, Iovine R, Negrini S, Robinson V, Kemper HC, Wells G, Tugwell P, Cranney A. Exercise for preventing and treating osteoporosis in postmenopausal women. Disponível em URL: http:/ / www.cochrane.org/ cochrane/revabstr/ab000333.htm. Acessado em 12 de abril de 2005.

24. Baumgartner RN, Koehler KM, Gallagher D, Romero L, Heymsfield SB, Ross RR, Garry PJ, Linderman RD. Epidemiology of sarcopenia among the elderly in New Mexico. Am J Epidemiol 1998; 147: 755-63.

25. Beverly MC, Rider TA, Evans MJ, Smith R. Local bone mineral response to brief exercise that stresses the skeleton. Br Med J 1989; 299: 233-35.

26. Kohrt WM, Ehsani AA, Birge Jr SJ. Effects of exercise involving predominately either joint reaction or ground reaction forces on BMD in older women. J Bone Miner Res 1997; 12: 1253-61.

27. Rubin CT, Lanyon LE. Regulation of bone formation by applied dynamic loads. J Bone Joint Surg 1984; 66A: 397 402

28. Nelson ME, Fiatarone MA, Morganti CM, Trice I, Greenber RA, Evans WJ. Effects of high intensity strength training on multiple risk factors for osteoporotic fractures. JAMA 1994; 272: 1909-14.

39. Gilsanz V, Boechat MI, Gilsanz R, Lord ML, Roe TF, Goodman WG. Gender differences in vertebral sizes in adults: biomechanical implications. Radiology 1994; 190: 678-82.

30. Sinaki M, Itoi E, Wahner HW, Wollan P, Gelzcer R, Mullan BP, Collins DA, Hodgson SF. Stronger back muscles reduce the incidence of vertebral fractures: a prospective 10 year follow-up of postmenopausal women. Bone 2002; 30(6): 836-41. 
31. Rutherford OM. Is there a role for exercise in the prevention of osteoporotic fractures? Br J Sports Med 1999; 33: 378-86.

32. Berard A, Bravo G, Gauthier P. Meta-analysis of the effectiveness of physical activity for the prevention of bone loss in postmenopausal women. Osteoporosis Int $1997 ; 7(4): 331-7$

33. Wolff I, van Croonenborg JJ, Kemper HC, Kostence PJ, Twisk JW. The effect of exercise training programs on bone mass: a meta-analysis of published controlled trials in pre- and postmenopausal women. Osteoporosis Int $1999 ; 9(1): 1-12$.
34. NIH - National Institute of Health - Declaracões de consensos: 111. Osteoporose-Prevenção, diagnóstico e tratamento. Disponível em URL: http:// consensus.nih.gov/ 2000/2000Osteoporosis111html.htm. Acessado em 4 de março de 2005.

recebido em: 04/05/06 versão final reapresentada em: 18/08/06 aprovado em: 20/09/06 
Anexo I - Discriminação dos estudos da base de dados do ACSM - BASE A

\begin{tabular}{|c|c|c|}
\hline $\begin{array}{l}\text { Estudos } \\
\text { Intervenções }\end{array}$ & \multicolumn{2}{|c|}{$\begin{array}{l}\text { Número participantes, País e Idade } \\
\text { Mensurações / Resultados (valor de P) }\end{array}$} \\
\hline 10 MESES & \multicolumn{2}{|c|}{108 ALEMANHA $56+/-9$ anos } \\
\hline grupo TR alta frequência: $>2$ a 4 vezes por semana & DMO vértebras lombares: & $+2.2 \%[P<0.001]$ \\
\hline grupo TR baixa frequência: 1 a 2 vezes por semana & DMO fêmur/Ward: & $+0.9 \%[\mathrm{P}<0.05]$ \\
\hline controles: não exercício ～DMO fêmur/colo: & \multicolumn{2}{|c|}{$-1.1 \%[P<0.05]$} \\
\hline
\end{tabular}

01.Kemmler W, Riedel H. The influence of a 10-month training program on risk factors in osteoporosis. Med Sci Sports Exerc 1999; 31(1) p191.

\section{MESES}

grupo TR: 60 a 90\% 1 RM (alta carga e alta repetição)

grupo TR + terapia hormonal

grupo caminhar

grupo caminhar + terapia hormonal

02. Humphries B, Newton RU, Bronks R, Marshall S, McBride J, Triplett-McBride T, Hakkinen K, Kraemer WJ, Humphries N. Effect of exercise intensity on bone density, strength, and calcium turnover in older women. Med Sci Sports Exer 2000; 32(6): 1043-1050.

\section{AUSTRÁLIA 45 - 65 anos}

força dinâmica (1 RM):peito/costas/coxas: $+25.8 \%$ e $+22.2 \%[P<0.05]$

força dinâmica (1 RM): peito/costas/coxas: $+25.4 \%$ e $+22.2 \%[\mathrm{P}<0.05]$

DMO vértebras lombares: $\quad-1.3 \%[\mathrm{P}<0.05]$

DMO vértebras lombares: $\quad-1.3 \%[\mathrm{P}<0.05]$

\section{MESES}

grupo TR alta carga: 80\% 1 RM, 3 séries, 8 repetições grupo TR alta repetição: 40\% 1 RM, 3 séries, 16 repetições controles: não exercício

${ }^{*} r=$ relação entre \% de mudança de osteocalcina e DMO

03. Bembem DA, Fetters NL, Bembem MG, Nabavi N, Koh ET. Musculoskeletal responses to high- and- low intensity resistance training in early postmenopausal women. Med Sci Sports Exerc 2000; 32(11):1949-57.

\section{MESES}

grupo TRP (progressivo): 2 a 3 séries, 8 a 10 repetições, 70 a $80 \% 1 R M$

$\begin{array}{ll}25 \text { EUA } 41 \mid-60 \text { anos } \\ \text { DMO quadril total : } & { }^{*} r=0.41[\mathrm{P}=0.048] \\ \text { DMO fêmur/trocanter: } & { }^{*} \mathrm{r}=0.42[\mathrm{P}=0.04]\end{array}$

\begin{tabular}{ll}
\multicolumn{1}{c}{19 EUA $44 \mid-\mathbf{6 8}$ anos } \\
potência: & $4.6+/-3.9 \%[P=0.002]$ \\
força dinâmica: & $1 \mathrm{RM}$ leg press: $+99.8 \%[\mathrm{P}=0.001]$ \\
& $1 \mathrm{RM}$ supino: $+34.4 \%[\mathrm{P}=0.001]$ \\
resistência absoluta & leg press: $+221 \%[\mathrm{P}=0.001]$ \\
& supino: $+112 \%[\mathrm{P}=0.001]$ \\
flexibilidade: & $+8.2 \%[\mathrm{P}=0.017]$
\end{tabular}

controles: não exercício - -

04. Adams KJ, Swank AM, Berning JM, Sevene-Adams PG, Barnard KL, Shimp-Bowerman J. Progressive strength training in sedentary, older African American women. Med Sci Sports Exerc 2001; 33(9):1567-76.

\begin{tabular}{|c|c|c|c|}
\hline & 14 MESES & 100 ALEMANHA 55.1 & - 3.4 anos \\
\hline grupo TR + calistênicos & & força dinâmica (1RM): & +15 para $+43 \%[\mathrm{P}<0.001]$ \\
\hline & & máxima força isométrica: & $+11 a+32 \%[P<0.001]$ \\
\hline & & DMO vértebras lombares: & $+1.3 \%[P<0.001]$ \\
\hline controles: não exercício & & DMO vértebras lombares: & $-1.2 \%[P<0.001]$ \\
\hline
\end{tabular}

05. Kemmler W, Engelke K, Lauber D, Weineck J, Hensen J, Kalender WA. Exercise effects on fitness and bone mineral density in early postmenopausal women: 1-year EFOPS results. Med Sci Sports Exerc 2002; 34(12):2115-23.

\section{MESES}

grupo TR alta carga e alta repetição: 2 séries, 6 a 8 repetições

70 a $80 \% 1 R M$

controles: não exercício

06. Cussler EC, Lohman TG, Going SB, Houtkooper LB, Metcalfe LL, Flint-Wagner HG, Harris RB, Teixeira PJ. Weight lifted in strength training predicts bone change in postmenopausal women. Med Sci Sports Exerc 2003; 35(1):10-17.

\section{EUA 44 |- 66 anos}

DMO fêmur/trocanter aumento: $\quad[\mathrm{P}<0.01]$

$\%$ mudança DMO todo corpo aumento: $\quad[\mathrm{P}<0.01]$

\begin{tabular}{|c|c|c|}
\hline 12 MESES & 233 EUA 40 & anos \\
\hline grupo TR + calistênicos & força dinâmica (1RM): & $+28 a+77 \%[P<0.001]$ \\
\hline grupo TR + terapia hormonal & força dinâmica (1 RM): & $+28 \mathrm{a}+77 \%[\mathrm{P}<0.001]$ \\
\hline controles: sem terapia hormonal - - & & \\
\hline controle + terapia hormonal - - & & \\
\hline $\begin{array}{l}\text { 07. Teixeira PJ, Going SB, Houtkooper LB, Metcalfe } \\
\text { postmenopausal women with and without horm }\end{array}$ & $\begin{array}{l}\text { Wagner HG, Cussler EC, Sarc } \\
\text { Sci Sports Exerc 2003; 35(4) }\end{array}$ & $\begin{array}{l}\text { ha LB, Lohman TG. Resistance training in } \\
5-62 \text {. }\end{array}$ \\
\hline 38 MESES & 78 ALEMANHA 55.1 & 3.3 anos \\
\hline grupo TRP (progressivo): 50 a 70 e 70 a $90 \% 1$ RM & DMO vértebras lombares: & $+0.7 \%$ (não significante) \\
\hline controles: não exercício & DMO vértebras lombares: & $-3.0 \%$ (não significante) \\
\hline
\end{tabular}


Anexo II - Discriminação dos estudos da base de dados da Biblioteca Cochrane - BASE B

\begin{tabular}{lcc}
\hline $\begin{array}{l}\text { Estudos } \\
\text { Intervenções }\end{array}$ & Duração & $\begin{array}{c}\text { Número participantes, País e Idade } \\
\text { Mensurações / Resultados [IC] }\end{array}$ \\
\hline $\begin{array}{l}\text { grupo TR + aeróbio } \\
\text { Controles: rotina qualquer de condicionamento físico } \\
\text { 01.Chow R, Harrison JE, Notarius C. Effect of two randomised exercise programmes on bone mass of health postmenopausal women. BMJ } \\
\text { 1987; 295:1441-44. }\end{array}$ & $\begin{array}{c}58 \text { CANADÁ } 50 \text {-70 anos } \\
\text { (mensurou índice de cálcio ósseo) / - - }\end{array}$
\end{tabular}
1987; 295:1441-44.

\begin{tabular}{lc}
\hline 24 MESES & 65 EUA - \\
grupo TR baixa carga $(30 \%$ 1RM) & DMO vértebras lombares IC $[+0.44$ a +4.57$]$
\end{tabular}

controles: não exercício

02. Sinaki M, Wahner HW, Offord KP, Hodgson SF. Efficacy of nonloading exercise in prevention of vertebral bone loss in postmenopausal women: a control trial. Mayo Clin Proc 1989; 64:762-69.

\begin{tabular}{|c|c|}
\hline $\begin{array}{l}\text { grupo TR para braços + aeróbios } \\
\text { grupo exercício + cálcio } \\
\text { grupo exercício + terapia hormonal } \\
\text { controles: não exercício }\end{array}$ & $\begin{array}{c}122 \text { AUSTRÁLIA - } \\
\text { DMO vértebras e antebraço/ não significante }\end{array}$ \\
\hline
\end{tabular}

\begin{tabular}{ll}
\hline grupo exercício alto impacto & 12 MESES EUA - \\
DMO vértebras lombares/não significante
\end{tabular}

grupo exercício baixo impacto

controles: não exercício

04. Grove KA, Londeree BR. Bone density in postmenopausal women: high impact vs low impact exercises. Med Sci Sports Exerc 1992; 24(11):1190-94.

\section{MESES}

grupo exercício calistênico + tablete placebo/dia

grupo exercício calistênico $+800 \mathrm{mg}$ cálcio/dia

controles: não exercício e $800 \mathrm{mg}$ cálcio/dia

não exercício e tablete placebo/dia

05. Lau EMC, Woo J, Leung PC, Swaminathan R, Leung D. The effects of calcium supplementation and exercise on bone density in elderly Chinese women. Osteo Int 1992; 2:168-173.

\section{MESES}

grupo TR: 70\% 1RM, 3 séries, 10 repetições

controles: não exercício

06. Smidt GL, Lin SY, O'Dwyer KD, Blanpied PR. The effect of high-intensity trunk exercise on bone mineral density of postmenopausal women. Spine 1992; 17:280.

\section{MESES}

grupo flexibilidade + caminhar moderado/intenso

controles: não exercício

07. Hatori M, Hasegawa A, Adachi H, Shinozaki A, Hayashi R, Okano H, Mizunuma H, Murata K. The effects of walking of the anaerobic threshold level on vertebral bone loss in postmenopausal women. Calcif Tissue Int 1993; 52:411-14.

\begin{tabular}{|c|c|}
\hline $\begin{array}{l}\qquad 12 \text { MESES } \\
\text { grupo esteira } 30 \text { minutos } \\
\text { grupo esteira } 45 \text { minutos } \\
\text { controles: não exercício + cálcio e vitamina D } \\
\text { 08. Martin D, Notelovitz M. Effects of aerobic training on bc } \\
\text { 8(8):931-36. } \\
\text { *estudo excluído por não estudar TR }\end{array}$ & $\begin{array}{l}\text { 76 EUA - } \\
\text { DMO vértebras e antebraço/não significante } \\
\text { nineral density of postmenopausal women. J Bone and Mineral Res 1993; }\end{array}$ \\
\hline $\begin{array}{l}12 \text { MESES } \\
\text { grupo força-psoas: } 60 \text { flexões com } 5 \mathrm{~kg} \text { sobre os joelhos } \\
\text { grupo força-deltóide: } 60 \text { abduções com } 1 \mathrm{~kg} \text { em cada mão } \\
2 \text { subgrupos assiduidade psoas e deltóide: compostos por } \\
\text { 09. Revel M, Mayoux-Benhamou MA, Rabourdin JP, Bac }\end{array}$ & $\begin{array}{l}\text { 78 FRANÇA - } \\
\text { \% mudança DMO todo corpo/não significante } \\
\text { heres que realizaram os exercícios assiduamente } \\
\text { i F, Roux C. One-year psoas training can prevent lumbar bone loss in } \\
\text { Tissue Int } 1993 ; 53: 307-11 \text {. }\end{array}$ \\
\hline $\begin{array}{l}\text { grupo TR alta carga } 12 \text { MESES } \\
\text { controles: não exercício } \\
\text { 10. Nelson ME, Fiatarone MA, Morganti CM, Trice I, Green } \\
\text { factors for osteoporotic fractures. JAMA 1994; } 272(24): 190\end{array}$ & $\begin{array}{l}\text { D0 EUA } \mathbf{5 0} \mid-\mathbf{7 0} \text { anos } \\
\text { DMO vértebras lombares IC }[+0.44 \mathrm{a}+4.57] \\
\text { DMO quadril total IC }[-8.5 \mathrm{a}+1.67] \text { não significante na meta-análise* } \\
\text { RA, Evans WJ. Effects of high-intensity strength training on multiple risk }\end{array}$ \\
\hline
\end{tabular}

\author{
50 CHINA 62 - 92 anos \\ $\%$ mudança $\mathrm{DMO} /$ não significante
}


Anexo II - Discriminação estudos da Biblioteca Cochrane - BASE B (continuação)

\begin{tabular}{lc}
\hline \multicolumn{1}{c}{$\mathbf{6 0}$ MESES } & \multicolumn{1}{c}{$\mathbf{1 4 6}$ ÁÚSTRIA $\mathbf{4 5} \mid-\mathbf{7 5}$ anos } \\
$\begin{array}{l}\text { grupo TR + flexibilidade } \\
\text { controles: não exercício }\end{array}$ & DMO punho IC [-3.21 a +2.65] não significante na meta-análise* \\
$\begin{array}{l}\text { 11. Preisinger E, Alacamlioglu Y, Pils K, Saradeth T, Scheneider } \\
\text { Rehabil 1995; T4(2):120-23. Therapeutic exercise in the prevention of bone loss. Am J Phys Med }\end{array}$
\end{tabular}
Rehabil 1995; 74(2):120-23.

-

grupo TR + aeróbios + calistênicos $\quad$ DMO vértebras lombares IC [+0.58 a +3.01$]$

DMO fêmur/colo e quadril IC [-1.18 a +1.03] não significante na meta-análise*

DMO punho IC [+0.71 a + 1.74] não significante na meta-análise*

controles: não exercício + tabletes placebo/dia

não exercício + $1 \mathrm{~g}$ cálcio/dia

não exercício + leite em pó com $1 \mathrm{~g}$ cálcio/dia

12. Prince R, Devine A, Dick I, Criddle A, Kerr D, Kent N, Price R, Randell A. The effects of calcium supplementation (milk powder or tablets) and exercise on bone density in postmenopausal women. J Bone \& Miner Res 1995; 10(7):1068-75.

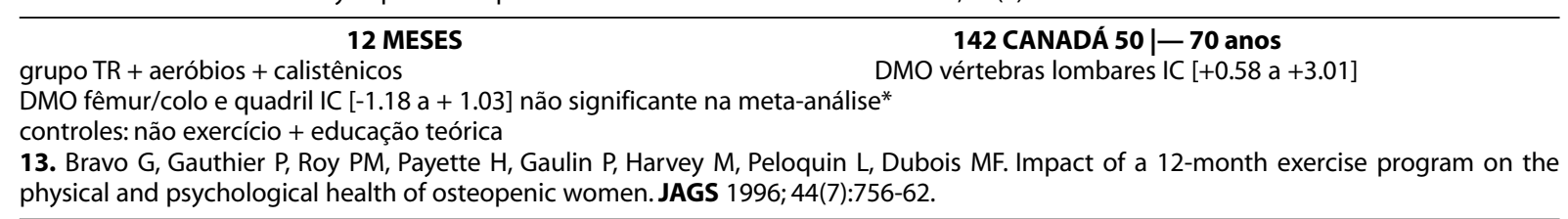
physical and psychological health of osteopenic women. JAGS 1996; 44(7):756-62.

- - 56 AUSTRÁLIA 40 - 70 anos

grupo TR + aeróbios + calistênicosDMO fêmur/colo e quadril IC [-1.18 a +1.03] não significante na meta-análise*

DMO punho IC [+0.71 a +1.74] não significante na meta-análise

controles: usou o lado do corpo que não praticou exercícios

14. Kerr D, Morton A, Dick I, Prince R. Exercise effects on bone mass in postmenopausal women is site-specific and load-dependent. J Bone \& Miner Res 1996; $11(2): 218-25$.

\section{MESES}

grupo aeróbio + flexibilidade + coordenação motora

controles: não exercício

15. Lord SR, Ward JA, Willians P, Zivanovic E. The effects of a community exercise program on fracture risk factors in older women. Osteo Int 1996; 6:361-67.

12 MESES

grupo TR alta carga

controles: não exercício

12 MESES older women. J Bone \& Miner Res 1996; 10(11):1788-95.

24 MESES $\quad$ 165 FRANÇA/ÁUSTRIA -

grupo TR + caminhar

protocolo: mais rápido ou difícil do que o usual

controles: não exercício

17. Ebrahim S, Thompson PW, Baskaran V, Evans K. Randomized placebo-controlled trial of brisk walking in the prevention of postmenopausal osteoporosis. Age and Aging 1997; 26:253-60.

\begin{tabular}{|c|c|}
\hline $\begin{array}{l}\qquad \mathbf{3 6} \text { MESES } \\
\text { grupo TR alta repetição e baixa carga } \\
\text { ativo no segundo e terceiro anos, inativo no primeiro ano } \\
\text { controles: ativo no primeiro ano e inativo nos segundo e te } \\
\text { 18. Mayoux-Benhamou MA, Bagheri F, Roux C, Auleley GR, } \\
\text { loss: A 3-years-follow-up study. Calcif Tissue Int 1997; } 60: 3\end{array}$ & $\begin{array}{l}\text { 51 FRANÇA } 55 \text { - } 61 \text { anos } \\
\text { DMO vértebras lombares IC [+0.44 a +4.57] } \\
\text { el M. Effect of Psoas Training on postmenopausal lumbar bone }\end{array}$ \\
\hline
\end{tabular}

*não significante na meta-análise: resultados considerados positivos isoladamente, mas não estatisticamente significantes quando integrados na meta-análise. 